Vietnam Journal of Mechanics, NCST of Vietnam Vol. 22, 2000, No I (1 - 10)

\title{
AN APPROXIMATE METHOD FOR ANALYSING NON-LINEAR SYSTEMS SUBJECT TO RANDOM EXCITATION
}

\author{
NGUYEN DONG ANH - NINH QUANG HAI \\ Institute of Mechanics, NCST of Vietnam
}

\begin{abstract}
A solution technique based on the representation of the response of the non-linear system by a polynomial of the response of the linearized system is presented. The relation between the original non-linear system and the linearized system is introduced by considering the so-called extended moment equations and their closed set is to be solved to determine unknowns. For the Vanderpol oscillator subject to white noise excitation, the technique gives good approximation to the response moments as well as the probability density function.
\end{abstract}

\section{Introduction}

Since all real engineering systems are, more or less, non-linear and for those systems the exact solutions are known only for a number of special cases, it is necessary to develop approximate techniques to determine the response of nonlinear systems under excitations. Several books examine approximate techniques for solving deterministic and / or random vibration problems, for instance, see $[1,2]$ and see $[3,4]$, respectively. This paper presents a solution technique based on the representation of the response of a non-linear system by a polynomial of the response of the linearized system. As the result of considering an original and the corresponding linear systems together, the extended moment equations are developed and their closed set is to be solved to determine unknowns. In [18] the application of the method to the Duffing system is presented. Herein, we continue our investigation on the Vanderpol oscillator subject to white noise excitation. It turns out that, the technique also gives good approximation to the response moments as well as the probability density function.

2. Extended moment equations and polynomial form for the system response

Consider the equation of motion of a single - degree - of - freedom mechanical 
system

$$
\ddot{z}+2 h \dot{z}+\beta z+f(z, \dot{z})=\sigma \dot{\xi}(t),
$$

where $h$ is a positive constant, $\beta$ is a constant, $f(z, \dot{z})$ is a polynomial function or approximated as a polynomial function of $z, \dot{z} ; \dot{\xi}(t)$ is a zero mean white noise with the autocorrelation and spectral density given, respectively, by

$$
R(\tau)=\langle\sigma \dot{\xi}(t) \sigma \dot{\xi}(t+\tau)\rangle=\sigma^{2} \delta(\tau) \quad \text { and } \quad S(\omega)=\frac{\sigma^{2}}{2 \pi}
$$

where $\delta(\tau)$ is Dirac delta function, \langle\rangle denotes the expectation operator. For sake of simplicity we suppose $\langle z\rangle=0$. Together with $(2.1)$ one considers the corresponding linearized equation

$$
\ddot{x}+2 \bar{h} \dot{x}+\bar{\omega}_{0}^{2} x=\sigma \dot{\xi}(t) .
$$

For an arbitrary up-to-second-order differentiable function $\varphi(z, \dot{z}, x, \dot{x})$ the extended moment equation corresponding to a stationary solution of (2.1), (2.2) has the form:

$$
\begin{aligned}
\left\langle\frac{\partial}{\partial z} \dot{z}\right\rangle & -\left\langle\frac{\partial \varphi}{\partial \dot{z}}(2 h \dot{z}+\beta z+f(z, \dot{z}))\right\rangle+\left\langle\frac{\partial \varphi}{\partial x} \dot{x}\right\rangle-\left\langle\frac{\partial \varphi}{\partial \dot{x}}\left(2 \bar{h} \dot{x}+\bar{\omega}_{0}^{2} x\right)\right\rangle+ \\
& +\frac{\sigma^{2}}{2}\left\{\left\langle\frac{\partial^{2} \varphi}{\partial \dot{z}^{2}}\right\rangle+2\left\langle\frac{\partial^{2} \varphi}{\partial \dot{z} \partial \dot{x}}\right\rangle+\left\langle\frac{\partial^{2} \varphi}{\partial \dot{x}^{2}}\right\rangle\right\}=0 .
\end{aligned}
$$

The equation (2.3) is derived from Fokker-Planck equation (e.g. see [3]). It should be noted that the conventional moment equation is derived from only the original non- linear equation (2.1). In order to investigate the correlation between the original variable $z$ and the linearized variable $x$, the equations (2.1) and (2.2) are considered together. Here, this attempt results in the extended moment equation (2.3).

Using (2.3) one can get, in the non-linear case, i.e., $f \neq 0$, a so-called infinite hierarchy of linear algebraic equations for the response moments in the sense that all finite sets of moment equations contain a number of involved unknown moments more than the number of equations (see [9-12]). Particularly, taking the 8 "lowest" polynomial functions $\varphi(z, \dot{z}, x, \dot{x})$, and using the well-known definition:

$$
m_{i j}=\left\langle z^{i} \dot{z}^{j}\right\rangle=E\left[z^{i} \dot{z}^{j}\right]
$$

one has following equations:

$$
\begin{aligned}
& \text { for } \varphi=\frac{1}{2} \dot{z}^{2}: \\
& \qquad-2 h m_{02}-\langle f(z, \dot{z}) \dot{z}\rangle+\frac{\sigma^{2}}{2}=0
\end{aligned}
$$


for $\varphi=z \dot{z}:$

$$
m_{02}-\beta m_{20}-\langle f(z, \dot{z}) z\rangle=0
$$

for $\varphi=\frac{1}{2} \dot{x}^{2}$ :

$$
2 \bar{h}\left\langle\dot{x}^{2}\right\rangle-\frac{\sigma^{2}}{2}=0
$$

for $\varphi=x \dot{x}$ :

$$
\bar{\omega}_{0}^{2}\left\langle x^{2}\right\rangle-\left\langle\dot{x}^{2}\right\rangle=0
$$

for $\varphi=z x$ :

$$
\langle x \dot{z}\rangle+\langle\dot{x} z\rangle=0
$$

for $\varphi=\dot{x} z$ :

$$
\langle\dot{x} \dot{z}\rangle-2 \bar{h}\langle\dot{x} z\rangle-\bar{\omega}_{0}^{2}\langle z x\rangle=0
$$

for $\varphi=\dot{x} \dot{z}$ :

$$
\sigma^{2}-2 h\langle\dot{x} \dot{z}\rangle-\beta\langle\dot{x} z\rangle-\langle\dot{x} f(z, \dot{z})\rangle-2 \bar{h}\langle\dot{x} \dot{z}\rangle-\bar{\omega}_{0}^{2}\langle\dot{z} x\rangle=0
$$

for $\varphi=x \dot{z}$ :

$$
\langle\dot{x} \dot{z}\rangle-2 h\langle x \dot{z}\rangle-\beta\langle z x\rangle-\langle x f(z, \dot{z})\rangle=0 .
$$

The moment equations (2.4)-(2.7) are conventional ones for separated moments of $z, \dot{z}$ and $x, \dot{x}$, while the moment equations (2.8)-(2.11) contain mixed moments of $z, \dot{z}, x, \dot{x}$. The equations (2.6), (2.7) are used separately to determine linearized coefficients:

$$
\bar{h}=\frac{\sigma^{2}}{4\left\langle\dot{x}^{2}\right\rangle} ; \quad \bar{\omega}_{0}^{2}=\frac{\left\langle\dot{x}^{2}\right\rangle}{\left\langle x^{2}\right\rangle} .
$$

It is seen that moment equations (2.4)-(2.11) have higher order moments. To close a set of moment equations one needs some additional relationships between moments. For instance, in Gaussian closure one puts $z(t)=x(t)$ and only 2 moment equations $(2.4),(2.5)$ can be satisfied, namely, they are used to find $\left\langle z^{2}\right\rangle$ and $\left\langle\dot{z}^{2}\right\rangle$. Thus, in Gaussian closure, in principle, only 2 equations from the hierarchy of moment equations can be satisfied. On the other hand, one might suppose that the accuracy of a closure technique would be better if more moment equations could be satisfied.

In the paper, we consider the following form of the system response

$$
z(t)=x(t)+\alpha_{1} x^{3}(t)+\alpha_{2} x^{5}(t) .
$$


Substituting (2.13) into (2.8)-(2.11) shows that the equations (2.8), (2.9) are satisfied for any $\alpha_{1}, \alpha_{2}$. Thus, finally one has 4 equations (2.4), (2.5), (2.10), (2.11) for determining 4 unknowns $\left\langle x^{2}\right\rangle,\left\langle\dot{x}^{2}\right\rangle, \alpha_{1}, \alpha_{2}$. It is noted that, the solution of the problem must satisfy not only the moment equations but also must preserve moment properties such as non-negativeness of even-order moments and satisfaction of Schwartz' inequality. The considered moment equations may yield a unique solution, multiple solutions or no solution. This matter should be investigated in other research. Particularly, for Gaussian closure we may see [13]. The probabilistic characteristics of $z(t)$ can be obtained from (2.13) if $x(t), \alpha_{1}, \alpha_{2}$ are known.

\section{Probability density function of response (PDF)}

Using the expression (2.13) one can easily calculate the PDF of the response process $z(t)$.' In fact, the PDF of $x$ is known as:

$$
p_{x}=\frac{\exp \left\{-x^{2} / 2 \sigma_{x}^{2}\right\}}{\sqrt{2 \pi \sigma_{x}^{2}}}
$$

The probability distribution function of $z$ is defined as

$$
F_{z}(y)=P\{-\infty \prec x \prec y\},
$$

where $P\{E\}$ denotes the probability of $E$. The PDF of $z$ can be found as follows

$$
\begin{aligned}
w_{z}(y) & =\lim _{\Delta y \rightarrow 0} \frac{F_{z}(y+\Delta y)-F_{z}(y)}{\Delta y}= \\
& =\lim _{\Delta y \rightarrow 0} \frac{P\{y \prec z \prec y+\Delta y\}}{\Delta y}=\lim _{\Delta y \rightarrow 0} \frac{1}{\Delta y} \int_{d} p_{x} d x,
\end{aligned}
$$

where the integration domain $d$ takes the form

$$
d=\{x: y<z<y+\Delta y\}=\left\{x: y<x+\alpha_{1} x^{3}+\alpha_{2} x^{5}<y+\Delta y\right\} .
$$

Thus, a numerical procedure to determine this function can be established.

\section{Power spectral density of response (PSD)}

The PSD is an important statistical characteristic of the stationary process. There are some investigations concerning with the PSD of the stationary response 
of non-linear stochastic systems (see [14-15]). Here using the expression (2.13) one can find the PSD of $z(t)$. The higher moments of a Gaussian random process are related to its second- order moments by known equations (see [16]). Applying the properties of Gaussian process we can express the second order autocorrelation function of $z(t)$ in terms of $R_{x}(\tau)$ :

$$
\begin{aligned}
R_{z}(\tau) & =\langle z(t) z(t+\tau)\rangle= \\
& =\left\langle\left[x(t)+\alpha_{1} x^{3}(t)+\alpha_{2} x^{5}(t)\right]\left[x(t+\tau)+\alpha_{1} x^{3}(t+\tau)+\alpha_{2} x^{5}(t+\tau)\right]\right\rangle= \\
& =\psi\left(R_{x}(\tau)\right),
\end{aligned}
$$

in which $R_{x}(\tau)$ (the second order autocorrelation function of $x(t)$ ) can be defined once the linearized equation (2.2) has been known (see[3]). The PSD of $z(t)$ is then defined as:

$$
S_{z}(\omega)=\frac{1}{\pi} \int_{0}^{+\infty} R_{z}(\tau) \cos \omega \tau d \tau .
$$

Based on the above-mentioned formulas, a numerical procedure for determining the PDS of $z(t)$ can be established.

\section{Vanderpol oscillator under white noise excitation}

To elucidate the proposed technique, in [18] we have investigated the Duffing oscillator under white noise

$$
\ddot{z}+2 h \dot{z}+\beta z+\varepsilon z^{3}=\sigma \dot{\xi}(t)
$$

in unimodal $(\beta=1)$ and bimodal $(\beta=-1)$ cases. Now, consider the Vanderpol system

$$
\ddot{z}-\varepsilon \dot{z}+z+\varepsilon \gamma z^{2} \dot{z}=\sigma \dot{\xi}(t)
$$

herein, one gets

$$
2 h=-\varepsilon, \beta=1, \quad \text { and } \quad f(z, \dot{z})=\varepsilon \gamma z^{2} \dot{z}
$$

A linearized equation corresponding to (5.1) is in the form

$$
\ddot{x}+2 \bar{h} \dot{x}+\bar{\omega}_{0}^{2} x=\sigma \dot{\xi}(t) .
$$

\subsection{Moment equations and polynomial form for solution}

For sake of simplicity and due to the limited capacity of our computer to determine $\left\langle x^{2}\right\rangle,\left\langle\dot{x}^{2}\right\rangle, \alpha_{1}$, the solution of the equation (5.1) is taken in the form: 


$$
z(t)=x(t)+\alpha x^{3}(t) .
$$

We consider 3 equations $(2.4),(2.5),(2.10)$ which now, respectively, become

$$
\begin{gathered}
\varepsilon m_{02}-\varepsilon \gamma m_{22}+\frac{\sigma^{2}}{2}=0, \\
m_{02}-m_{20}-\varepsilon \gamma m_{31}=0, \\
\sigma^{2}+\varepsilon\langle\dot{x} \dot{z}\rangle-\langle\dot{x} z\rangle-\varepsilon \gamma\left\langle\dot{x} z^{2} \dot{z}\right\rangle-2 \bar{h}\langle\dot{x} \dot{z}\rangle-\bar{\omega}_{0}^{2}\langle\dot{z} x\rangle=0 .
\end{gathered}
$$

It follows from (5.4)

$$
\begin{aligned}
& \langle\dot{x} z\rangle=0, \\
& \langle\dot{z} x\rangle=0, \\
& m_{31}=0 .
\end{aligned}
$$

Using (5.8), (2.12) one can turn the equations (5.6)-(5.7) inito the following forms

$$
\begin{gathered}
m_{02}-m_{20}=0 \\
\sigma^{2}+\varepsilon\langle\dot{x} \dot{z}\rangle-\varepsilon \gamma\left\langle\dot{x} z^{2} \dot{z}\right\rangle-\frac{\sigma^{2}}{2\left\langle\dot{x}^{2}\right\rangle}\langle\dot{x} \dot{z}\rangle=0 .
\end{gathered}
$$

Denoting $y=\left\langle x^{2}\right\rangle$, if follows from (5.4)

$$
\begin{gathered}
m_{20}=y+6 \alpha y^{2}+15 \alpha^{2} y^{3} \\
m_{02}=\left\langle\dot{x}^{2}\right\rangle\left[1+6 \alpha y+27 \alpha^{2} y^{2}\right] \\
m_{22}=\left\langle\dot{x}^{2}\right\rangle\left[y+24 \alpha y^{2}+330 \alpha^{2} y^{3}+2520 \alpha^{3} y^{4}+8505 \alpha^{4} y^{5}\right] \\
\langle\dot{x} \dot{z}\rangle=\left\langle\dot{x}^{2}\right\rangle(1+3 \alpha y) \\
\langle x z\rangle=y+3 \alpha y^{2} \\
\left\langle\dot{x} z^{2} \dot{z}\right\rangle=\left\langle\dot{x}^{2}\right\rangle\left[y+15 \alpha y^{2}+105 \alpha^{2} y^{3}+315 \alpha^{3} y^{4}\right] .
\end{gathered}
$$

From $(5.9),(5.11)$ and $(5.12)$ one gets

$$
\left\langle\dot{x}^{2}\right\rangle=\frac{\left[y+6 \alpha y^{2}+15 \alpha^{2} y^{3}\right]}{\left[1+6 \alpha y+27 \alpha^{2} y^{2}\right]} .
$$

Using (5.12) - (5.17) after some calculation, it follows from (5.5) and (5.10)

$$
\begin{aligned}
& \sigma^{2}+2 \varepsilon y+6 \sigma^{2} \alpha y+24 \varepsilon \alpha y^{2}-2 \varepsilon \gamma y^{2}+27 \sigma^{2} \alpha^{2} y^{2}+156 \varepsilon \alpha^{2} y^{3}-60 \varepsilon \gamma \alpha y^{3}+ \\
& +504 \varepsilon \alpha^{3} y^{4}-978 \varepsilon \gamma \alpha^{2} y^{4}+810 \varepsilon \alpha^{4} y^{5}-9720 \varepsilon \gamma \alpha^{3} y^{5}-57150 \varepsilon \gamma \alpha^{4} y^{6}- \\
& -177660 \varepsilon \gamma \alpha^{5} y^{7}-255150 \varepsilon \gamma \alpha^{6} y^{8}=0
\end{aligned}
$$




$$
\begin{aligned}
& \sigma^{2}+2 \varepsilon y+3 \sigma^{2} \alpha y+18 \varepsilon \alpha y^{2}-2 \varepsilon \gamma y^{2}+9 \sigma^{2} \alpha^{2} y^{2}+66 \varepsilon \alpha^{2} y^{3}- \\
& -42 \varepsilon \gamma \alpha y^{3}-81 \sigma^{2} \alpha^{3} y^{3}+90 \varepsilon \alpha^{3} y^{4}-420 \varepsilon \gamma \alpha^{2} y^{4}- \\
& -2340 \varepsilon \gamma \alpha^{3} y^{5}-6930 \varepsilon \gamma \alpha^{4} y^{6}-9450 \varepsilon \gamma \alpha^{5} y^{7}=0
\end{aligned}
$$

for 2 unknowns: $y$ and $\alpha$. For these equations, the moment properties such as non-negativeness of even-order moments and satisfaction of Schwartz' inequality are used to exclude some extraneous solutions. Finally, the second moment $m_{20}$ (or $\left\langle z^{2}\right\rangle$ ) can be calculated from (5.11). The results $\left\langle z^{2}\right\rangle$ obtained by the proposed procedure and that obtained by Gaussian closure with the values $\gamma=10, \varepsilon=0.2$ are compared in Table 1 for different values of $\sigma^{2}$. It is seen that the proposed solutions $\left\langle z^{2}\right\rangle$ are much closer to the stimulation solutions $\left\langle z^{2}\right\rangle_{M C}[3]$ than the

\begin{tabular}{|c|c|c|c|c|c|c|}
\hline $\mathrm{N}^{\circ}$ & $\sigma^{2}$ & $\left\langle z^{2}\right\rangle_{M C}$ & $=\left\langle x^{2}\right\rangle_{G}$ & $\alpha$ & $\left\langle x^{2}\right\rangle$ & $\left\langle z^{2}\right\rangle_{L}$ \\
\hline 1 & 0.02 & 0.2080 & $0.137(-34 \%)$ & -0.2595 & 0.3632 & $0.2062(-0.9 \%)$ \\
\hline 2 & 0.2 & 0.3600 & $0.279(-22 \%)$ & -0.1329 & 0.6583 & $0.3883(7.9 \%)$ \\
\hline 3 & 1.0 & 0.7325 & $0.552(-25 \%)$ & -0.0679 & 1.2353 & $0.7437(1.5 \%)$ \\
\hline 4 & 2.0 & 1.0310 & $0.759(-26 \%)$ & -0.0496 & 1.6725 & $1.0129(-1.8 \%)$ \\
\hline 5 & 4.0 & 1.4540 & $1.051(-28 \%)$ & -0.0358 & 2.2927 & $1.3945(-4.1 \%)$ \\
\hline
\end{tabular}
solutions obtained by Gaussian closure $\left\langle x^{2}\right\rangle_{G}$.

Table 1. Approximate mean squares of response of the Vanderpol system

\subsection{Probability density function of response}

The PDF of the response of the bimodal Duffing oscillator is investigated, for example, in [17]. Herein for the Vanderpol system, the PDF is calculated from (3.3) where the formula (3.4) takes the form:

$$
d=\left\{x: y<z=x+\alpha x^{3}<y+\Delta y\right\}
$$

and $\sigma_{x}^{2}=\left\langle x^{2}\right\rangle$ is found from (5.18)-(5.19).

The PDF in Gaussian closure takes the form

$$
p_{G}(x)=\frac{\exp \left\{-x^{2} / 2\left\langle x^{2}\right\rangle_{G}\right\}}{\sqrt{2 \pi\left\langle x^{2}\right\rangle_{G}}}
$$

where

$$
\left\langle x^{2}\right\rangle_{G}=\frac{\left(\varepsilon+\sqrt{\varepsilon^{2}+2 \varepsilon \gamma \sigma^{2}}\right)}{2 \varepsilon \gamma}
$$


The graphs of probability density function (PDF) obtained by the proposed procedure and by the Gaussian closure for $\sigma^{2}=0.2$ and $\sigma^{2}=4$ are shown in Figures 1-a and 1-b. It can be seen that, the technique gives better description of the PDF.

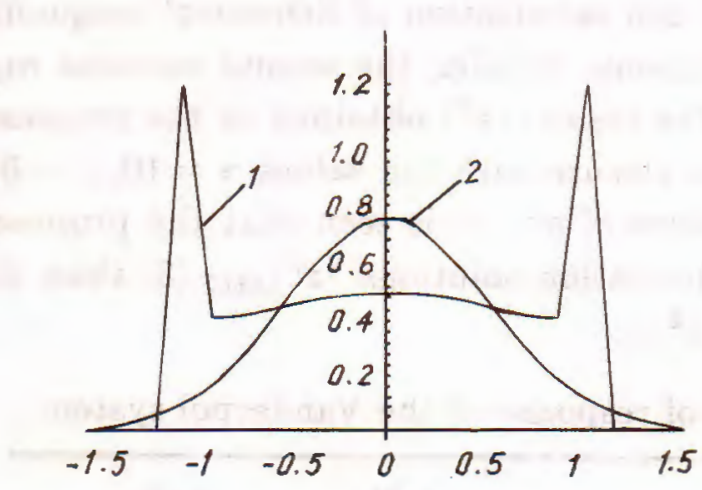

Fig. $1 a \gamma=10, \varepsilon=0.2, \sigma^{2}=0.2$

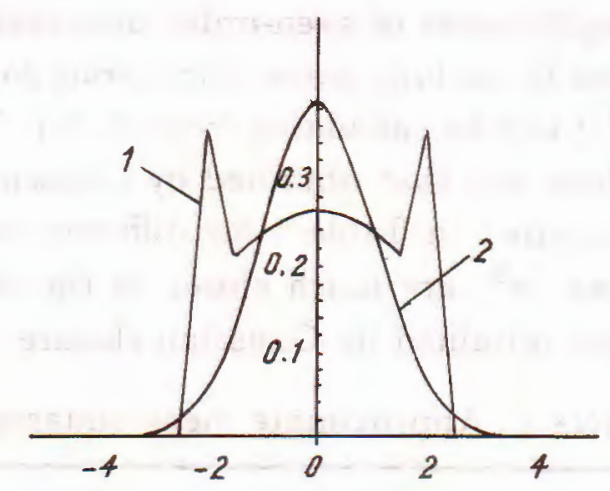

Fig. $16 \gamma=10, \varepsilon=0.2, \sigma^{2}=4$

1-The PDF of proposed method, 2-The PDF of Gaussian closure

\section{Conclusion}

The main idea of the proposed technique is to represent the system response by a polynomial of Gaussian process. Besides, the original and the corresponding linearized systems are considered together. As the result; the so-called extended moment equation is established. Based on this equation, the correlation between the original variable $z$ and the linearized variable $x$ is shown, and the application also is simplified. Thus, a possible way to determine the polynomial coefficients and the Gaussian process can be derived. Furthermore, the technique is quite simple since it can use properties of the Gaussian process although the calculations are more complicated than the Gaussian closure. The proposed method has been effectively applied to the Duffing and Vanderpol oscillators. However, the PSD of the Vanderpol system needs to be investigated, the technique should be tested for other non-linear systems and some related questions may occur: Which set of extended moment equations and which form of polynomials should be chosen to get a better approximate solution?

The research has been supported by a grant of the Fundamental Research Program in Natural Sciences. 


\section{REFERENCES}

1. Bogoliubov N. N. and Mitroposkii Iu. A. Asymptotic Methods in the Theory of Nonlinear Oscillations, Nauka, Moscow, (Russian), 1961.

2. Nayfeh A. H. Perturbation Methods. John Wiley and sons, 1973.

3. Roberts J. B. and Spanos P. D. Random Vibration and Stochastic Linearization, John Wiley and Sons, 1990.

4. Lin Y. K., Cai G. Q. Probabilistic Structural Dynamics. Advanced theory and Applications, Mc Graw-Hill, Inc, 1995.

5. Korn G. A., Korn T. M. Mathematical Handbook, Mc Graw-Hill, 1968.

6. Winterstain S. R. "Non-linear vibration models for extremes and fatigue", Journal of Engineering Mechanics, ASCE, 114, No.10, (1988), 1772-1790.

7. Grigoriu M. Applied Non-Gaussian Processes, PTR Prentice Hall, Englewood Cliffs, NJ, 1995.

8. Lutes L. D. and Sarkani S. Stochastic Analysis of Structures and Mechanics, Prentice-Hall, Englewood Cliffs, NJ, 1997.

9. Crandall S. H. Non-Gaussian closure for random of non-linear oscillators. Int. J. Non-linear Mech., 15 (1980), 303-313.

10. Ibrahim R. A., Soundararajan A. and Heo H. Stochastic response of non-linear dynamic systems based on non - Gaussian closure. Trans. Of the ASME, J. of Appl. Mech. 52 (1985), 965 - 970.

11. Wojtkiewicz S. F., Spencer Jr. B. F., Bergman L. A. New insights on the application of moment closure methods to non-linear stochastic systems. In Proc. of IUTAM Symposium on Non-Linear Stochastic Dynamics, eds Naess A. and S.. Krenk, Kluwer, (1996), $479-488$.

12. Anh N. D., Schiehlen W. An approach to the problem of closure in the nonlinear stochastic Mechanics. Int. J. of Mechanics, 29 (1994), 109 - 123.

13. Spanos P. D. and Iwan W. D. On the existence and uniqueness of solutions generated by Equivalent linearization, "International Journal of Nonlinear Mechanics, 13 (1978), 71-78.

14. Miles, R. N. An approximate solution for the spectral response of Duffing's oscillator with random input. Journal of Sound and Vibration 132 (1), (1989), 43-49.

15. Bouc R. The power spectral density of response for strongly non-linear random oscillator. Journal of Sound and Vibration, 175 (3), (1994), 317-331. 
16. Robson J. D. A simplified quasi-Gaussian random process model based on non-linearity. Journal of Sound and Vibration 76 (2), (1981), 169-177.

17. Alaoui Ismaili M. \& Bernard P. Asymptotic analysis and linearization of the randomly perturbed Two-Well Duffing oscillator. Prob. Eng. Mech. 12, No 3, (1997), 171-178.

18. Nguyen Dong Anh and Ninh Quang Hai. A Technique for solving non-linear systems subject to random excitation. In Proc. of IUTAM Symposium on recent developments in non-linear oscillations of Mechanical systems March 2-5, 1999, eds Naess A. and S.. Krenk, Kluwer.

Received November 2S, 1999

\section{PHƯONG PHÅP GẦN ĐÚNG ĐỂ PHÂN TÍCH CÁC HỆ PHI TUYẾN CHỊU KİCH ĐộNG NGẪU NHIÊN}

Bài báo trình bày một kỹ thuật giải dựa trên việc biểu diễn đáp ứng của hệ phi tuyến chịu kích động ngẫu nhiên bằng đa thức của đáp ứng Gauss của hệ tuyến tính hóa tương ứng. Mối quan hệ giữa phương trình phi tuyến gốc và phương trình tuyến tính tương ứng được thể hiện qua mối tương quan mô men giữa các đáp ứng thông qua việc xây dựng cái gọi là "các phương trình mô men suy rộng". Tập hợp đóng của các phương trình này được giải để xác định các ân cần tìm. Khi áp dụng cho hệ dao động Vanderpol chịu kích động ồn trăng, phương pháp này cho kết quả tốt về mô men đáp ứng lẫin hàm mật độ xác suất (PFD). Hơn thế, nó mô tả được đặc trưng khổng-Gawss của đáp ứng (thể hiện được hiệu ứng phi tuyến) trên dáng điệu của hàm $\mathrm{PFD}$ này.

Institute of Mechanics

264 Doi Can, Hanoi Vietnam 